\title{
Whole exome sequencing and methylation-specific multiplex ligation-dependent probe amplification applied to identify Angelman syndrome due to paternal uniparental disomy in two unrelated patients
}

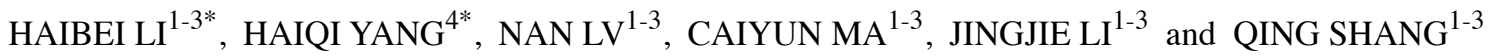 \\ ${ }^{1}$ Department of Pediatrics, Children's Hospital Affiliated to Zhengzhou University; ${ }^{2}$ Department of Pediatrics, \\ Henan Children's Hospital; ${ }^{3}$ Department of Pediatrics, Zhengzhou Children's Hospital, Zhengzhou, Henan 450053; \\ ${ }^{4}$ Aegicare (Shenzhen) Technology Co., Ltd., Shenzhen, Guangdong 518060, P.R. China
}

Received May 31, 2018; Accepted January 17, 2019

DOI: $10.3892 / \mathrm{mmr} .2019 .10339$

\begin{abstract}
Angelman syndrome (AS) is a congenital neurodevelopmental disorder typically occurring due to functional defects of the $U B E 3 A$ gene caused by uniparental disomy (UPD), translocation or single gene mutation. UBE3A gene exhibits imprinting expression, and only maternal inherited alleles express functional UBE3A protein in the brain. The common method to diagnose AS is single nucleotide polymorphism array or methylation-specific multiplex ligation-dependent probe amplification (MS-MLPA). In recent years, whole exome sequencing (WES) has been increasingly used in the genetic diagnosis of a variety of indications, exhibiting great advantages as a comprehensive and unbiased testing method. In the present study, the cases of two unrelated patients with Robertsonian-like translocation in chromosome 15 , namely $45, \mathrm{XX}, \operatorname{der}(15 ; 15)$ (q10;q10) and 45,XY,der(15;15)(q10;q10), are reported. The first case was diagnosed with AS by WES and validated by Sanger sequencing. In contrast to $42.84 \%$ homozygous variants on all chromosomes, $92.69 \%$ homozygosity variants were observed on chromosome 15. A homozygous stretch identifier was applied and identified a homozygous region across the entire chromosome 15. Sanger sequencing was used to further determine the subtype and confirm that two homozygous variants on chromosome 15 with low allele frequency $(<0.01)$ were derived only from the father and not from the mother, thereby indicating a paternal UPD case, classified as isodisomy.
\end{abstract}

Correspondence to: Dr Qing Shang, Department of Pediatrics, Children's Hospital Affiliated to Zhengzhou University, 255 Gangdu Street, Zhengzhou, Henan 450053, P.R. China

E-mail: sqing1965@163.com

${ }^{*}$ Contributed equally

Key words: Angelman syndrome, uniparental disomy, whole exome sequencing, isodisomy, Sanger sequencing
MS-MLPA results of the other AS patient with the same karyotype indicated that he had a high possibility of paternal UPD at chromosome 15. Taken together, the current study suggested the potential application of WES in detecting and facilitating the diagnosis of UPD.

\section{Introduction}

Dysfunction of ubiquitin-protein ligase E3A (encoded by $U B E 3 A$ gene) is the major cause of Angelman syndrome (AS), which is a congenital neurodevelopmental disorder presenting a series of clinical characteristics associated with neurological damage, including cognitive disability, cognitive delay, unconscious limb tremor or seizures, as well as inappropriate emotional response and behavior (1). UBE3A is located at chromosome 15 , encoding an enzyme that participates in the protein degradation process within cells. $U B E 3 A$ exhibits imprinted gene expression, that is, only maternally inherited $U B E 3 A$ can express functional proteins in the brain $(2,3)$. This functional defect results in the accumulation of several proteins in the brain that interfere with neuron development (4). It has been reported that single nucleotide variations (SNVs), maternal deletion of chromosome 15q11.2-q13 region or paternal chromosome 15 uniparental disomy (UPD) can affect the expression or function of $U B E 3 A$ gene and further lead to AS (5).

The majority of reported AS cases are caused by maternal deletion, whereas few cases are due to paternal UPD, where both copies of a chromosome are inherited from the father. Studies have demonstrated that approximately $2-5 \%$ of the AS patients were diagnosed because they had the only paternal allele of the $U B E 3 A$ gene, which is specifically silenced in the developing brain $(6,7)$. Heterodisomy and isodisomy are the two types of UPD. The former usually occurs when the diploid from one parent fails to separate into chromatids during meiosis I, while the latter occurs when the chromatids fail to separate during meiosis II (8). In consequence, isodisomy is when both chromosomes are from one parent and heterodisomy is where the two chromosomes are different copies of the same 
chromosome. UPD due to a parental Robertsonian translocation $(\mathrm{ROB})$ is rare, with a risk of $0.6-0.8 \%$ for non-homologous ROBs (9). However, isodisomy is a more frequent situation that can result from meiotic or mitotic duplication of one parental chromosome and may involve isochromosomes (10).

Several genetic tests have been used for the screening of UPD, such as microsatellite analysis, single nucleotide polymorphism (SNP)-based array comparative genomic hybridization and multiplex ligation-dependent probe amplification (MLPA). Recently, whole exome sequencing (WES) has been reported to be another feasible approach to determine UPD and assist the analysis of disease-causing variants (11-13). Exons are protein-coding regions consisting of only $1-2 \%$ of the genome, but contributing to almost $85 \%$ of reported disease-causing genes. Isodisomy can cause homozygosity in the autosomal recessive traits, which is detectable by homozygosity mapping tools, such as homozygous stretch identifier (HomSI) (14). Furthermore, the primary diagnosis of paternal isodisomy can be further validated by Sanger-based segregation analysis (11).

In the present study, the identification of two patients with an abnormal karyotype of balanced translocations on chromosome 15 is reported. For patient 1, variants were investigated using WES, and a higher homozygous rate was observed on chromosome 15 as compared with all chromosomes (92.69 vs. 42.84\%), indicating a high potential UPD. Chromosomes were mapped by HomSI (14) to further identify the homozygous regions on chromosome 15 . Next, Sanger sequencing validation indicated that two homozygous variants (STARD9, NM_020759.2:c.4169C>T; and TTBK2, NM_173500.3:c.2926C>T) with low frequency in Exome Aggregation Consortium (ExAC) databases were inherited from the father, which strongly suggests the paternal isodisomy type of UPD. In addition, AS was diagnosed in patient 2 by MS-MLPA, who exhibited paternal UPD on the 15q11-13 region.

\section{Patients and methods}

Ethical considerations. The present study was approved by the Ethics Committees of the Children's Hospital Affiliated to Zhengzhou University (Zhengzhou, China). The two cases involved patients admitted at the Children's Hospital Affiliated to Zhengzhou University, and written informed consents were obtained from all participating individuals. The parents of the studied patients declared that they were non-consanguineous and had no family history of congenital anomalies.

\section{Patient details}

Patient 1. Patient 1 was a 3 -year-old female with complex developmental issues. The patient had non-consanguineous parents and a healthy 12-year-old brother, and was born at 37 weeks of gestation by Caesarian section with a birth weight of $2.7 \mathrm{~kg}$. She sat at 12 months and stood unsupported for 1 minute or walk a few steps at 32 months. Absence of speech was reported, and the patient was only able to say 'mama'. The patient was shy, but always had a smiling face, and was able to signal 'goodbye' with gestures and recognize common objects. A seizure was first reported at the age of 2.5 years, and the anticonvulsant magnesium valproate was administered thereafter. In addition, the patient exhibited microcephaly, with a head circumference of $44 \mathrm{~cm}$. Genetic detection indicated a 45,XX,der(15;15)(q10;q10) karyotype (Fig. 1A). An electroencephalogram (EEG) indicated a moderate diffuse abnormality, while brain magnetic resonance imaging (MRI) detected a Rathke's cleft cyst. The blood acylcarnitine and amino acid profiles of the patient were normal. Elevated valproic acid was determined in the patient's urine, which may be the result of the anticonvulsant treatment.

Patient 2. Patient 2 was a 3.5-year-old male with a karyotype of 45,XY,der(15;15)(q10;q10) (Fig. 1B). The patient was born at 37 weeks of gestation by Caesarian section due to the umbilical cord being wrapped around the neck for two weeks, and weighed $3.7 \mathrm{~kg}$ at birth. His mother was 36 years old and the patient was her fifth pregnancy. The patient sat and began crawling at 8 months, and walked at the age of 1 year and 8 months. At 3 years old, the patient was able to run, go up and down stairs independently, and jump with both feet, but was unable to stand on a single leg. The subject was unable to speak, with the exception of unconsciously articulating 'mama' and 'nine'. He was able to understand certain simple commands and follow the instructions to finish specific actions. The patient had a happy smiling face and quick temper, as well as spaced teeth, bilateral inverted nipples and a micropenis. There was no history of seizures, and normal head development with a head circumference of $50 \mathrm{~cm}$ was observed. A brain MRI scan was normal. Blood acylcarnitine and amino acid profiles indicated a low level of serum acylcarnitine, which was treated with L-carnitine. Urinary organic acid levels were found to be normal.

DNA extraction and library construction. Genomic DNA was extracted from a 300- $\mu 1$ peripheral blood sample using a RelaxGene Blood DNA system (Tiangen Biotech Co., Ltd., Beijing China). Its purity and quantity were determined with a Nanodrop 2000 spectrophotometer and an Invitrogen Qubit $^{\circledR} 3.0$ fluorometer (Thermo Fisher Scientific, Inc., Waltham, MA, USA). The hybrid selection of genomic DNA was performed using Agilent SureSelect Human All Exon Kit v6 (Agilent Technologies, Inc., Santa Clara, CA, USA). DNA libraries were purified by magnetic beads (AMPrure XP beads; Beckman Coulter, Inc., Brea, CA, USA). Coding regions and intron/exon boundaries were enriched, and sample quality control was conducted by an Agilent 2100 Bioanalyzer system (Agilent Technologies, Inc.). The extracted DNA was stored at $-20^{\circ} \mathrm{C}$ and prepared for sequencing.

WES and data analysis. Samples were sequenced using the Illumina HiSeq X Ten platform (Illumina, Inc., San Diego, CA, USA). The results were analyzed and annotated by an in-house pipeline. Briefly, raw reads were preprocessed to remove reads with low quality or adaptors. Read alignment was performed using the Burrows-Wheeler Aligner tool (version 0.7.17) with default parameters against the human genome assembly hg19 (GRCh37) as previously described (15). The generated bam file was sorted by SAMtools (16). Genome Analysis Toolkit (GATK; https://software.broadinstitute.org/gatk/) was performed to detect SNVs and indels ( $<50 \mathrm{bp})$, and CNVkit (17) was applied to detect the copy number variations 
A

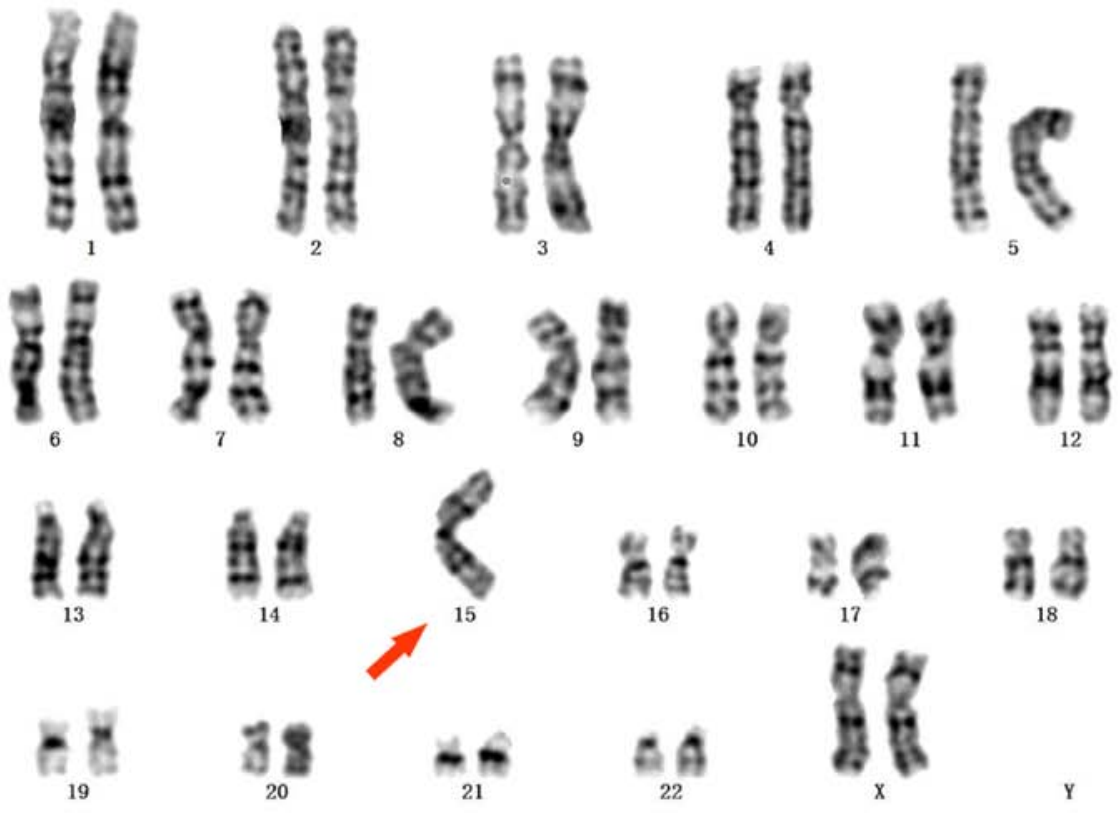

B
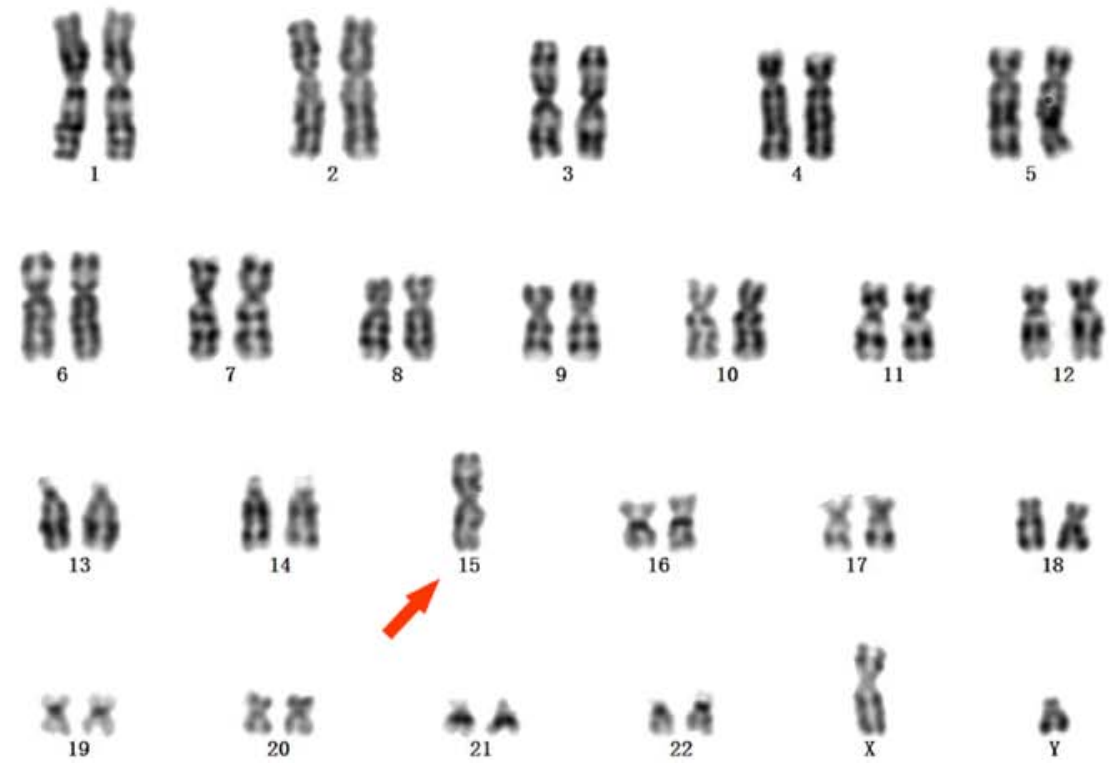

Figure 1. Karyotype of patients 1 and 2. (A) Patient 1 exhibited a karyotype of 45,XX,der(15;15)(q10;q10). (B) Patient 2 exhibited a karyotype of 45,XY,der(15;15) (q10;q10). The translocated chromosome is indicated by the arrow.

(CNVs). Next, the Variant Effect Predictor (18) was employed to annotate SNVs, indels and CNVs. With regard to possible effects on protein function, variants were evaluated by two widely used prediction tools, including Sorting Intolerant from Tolerant (SIFT) (19) and Polymorphism Phenotyping version 2 (PolyPhen-2) (20). For calculating the homozygous ratio with SNV data, the following rules were adopted: i) SNPs with a depth of $<20 x$ were removed; ii) only SNPs with a variant allele frequency between 0.2 and 0.8 were considered to be heterozygous.

Prioritization of candidate variants associated with disease phenotypes. Based on the aforementioned variant annotations, a series of prioritization strategies were applied to select candidate variants associated with clinical phenotypes. Detailed steps were as follows: i) Variants with minor allele frequency (MAF) of $>0.05$ according to public databases were excluded; ii) variants with no damaging results presented in any protein function predicted by SIFT and Polyphen 2 were excluded; and iii) variants described as benign or likely benign in ClinVar (18), and not as disease-causing in HGMD (21) were excluded. A candidate list of $\sim 100$ variants was obtained by implementing the aforementioned three-step prioritization strategy.

Homozygosity mapping. Homozygosity mapping was conducted with HomSI from next-generation sequencing data. In order to generate input files compatible with HomSI, VCFtools (22) was used to merge the variant call format (vcf) files of patient 1 and three healthy control individuals. Three individuals were all healthy individuals. Control 1 and 3 were male, while control 2 was a female. 
Paternal isodisomy confirmation. Two homozygous variants with high quality (read depth, $\geq 10$; genotype quality, $\geq 75)$ and rarity (1000 Genomes Project MAF $\leq 0.001$ (http://browser.1000genomes.org) and ExAC $\leq 0.001$ (http://exac.broadinstitute.org) were selected among the possible UPD sites (11). The genomic DNA were extracted from the peripheral blood sample of Patientl parents, which used as a template for Polymerase chain reaction (PCR). PCR was performed using a Bio-Rad thermal cycler (Bio-Rad Laboratories, Inc., Hercules, CA, USA). The forward and reverse primers for gene STARD9 (NM_020759.2:c.4169C $>$ T) were 5'-CCATTGTGAGCAGGCTGAAT-3' and 5'-ACGCTA GAATTGTGGGATGC-3', respectively. The forward and reverse primers for gene TTBK2 (NM_173500.3:c.2926C>T) were 5'-GAGAATGAACATGGTGCCCC-3' and 5'-AAGGGA GCAGGAACAGTAGC-3', respectively. The PCR conditions were: Denaturation at $95^{\circ} \mathrm{C}$ for $5 \mathrm{~min}$, followed by 10 cycles of $95^{\circ} \mathrm{C}$ for $30 \mathrm{sec}, 61^{\circ} \mathrm{C}$ for $30 \mathrm{sec}$ and $72^{\circ} \mathrm{C}$ for $30 \mathrm{sec}, 32 \mathrm{cycles}$ of $95^{\circ} \mathrm{C}$ for $30 \mathrm{sec}, 58^{\circ} \mathrm{C}$ for $30 \mathrm{sec}$ and $72^{\circ} \mathrm{C}$ for $30 \mathrm{sec}$, and a final elongation step at $72^{\circ} \mathrm{C}$ for $5 \mathrm{~min}$. PCR products were separated by $1 \%$ agarose gel electrophoresis and then purified by a QIAquick Gel Extraction kit (Qiagen, Inc., Valencia, CA, USA). Subsequently, the products were sequenced by the $3730 \mathrm{xl}$ DNA Analyzer Sequencing Standards, BigDye ${ }^{\mathrm{TM}}$ Terminator version 3.1 (Applied Biosystems; Thermo Fisher Scientific, Inc.) and 3730xl DNA Analyzer (Applied Biosystems; Thermo Fisher Scientific, Inc.).

MS-MLPA. MS-MLPA was conducted using a commercial MLPA probe kit, namely the SALSA MS-MLPA ME028-B1 Prader-Willi Syndrome (PWS)/AS probemix, following the protocols of the manufacturer (MRC-Holland, Amsterdam, Netherlands). The probemix contains 32 MLPA probes specific for sequences in or near the PWS/AS critical region of chromosome $15 \mathrm{q} 11$, which can be applied to detect the CNVs in this region. Five of these probes are designed for specific imprinted gene sequences and contain a recognition site for the methylation-sensitive HhaI enzyme. In addition, six probes are designed to detect indels and variations in $U B E 3 A$. In order to analyze the results, 14 reference probes were located outside the targeted region. Amplicons were identified by capillary electrophoresis, and the results were analyzed using Coffalyser.Net (MRC-Holland; https://support. mlpa.com/kb/coffalyser-net).

\section{Results}

Variant analysis by WES of patient 1. Exome sequencing yielded a total of $184,605,772$ reads with $91.52 \%$ above the Q30 score. Variants were annotated for population frequency and functional predictions. Across all chromosomes, $42.84 \%$ of the detected variants exhibited homozygosity, which was notably up to be $92.69 \%$ on chromosome 15 homozygotic variants (the remaining non-homozygous variants were mostly in intergenic regions; Table I).

With an MAF cut-off at 0.01 , a total of 18,176 variants were identified in whole exome regions, 470 of which were on chromosome 15 . Among all variants on chromosome 15 , there were 228 non-coding variants, 21 missense variants, 3 synonymous variants, 3 splice site variants, 1 indels, 2 frameshifts and 2 stop gained variants, whereas no stop-lost and start-lost variants were detected. Similar results with that of all variants were detected for the homozygous variant ratio, with a value of $39.66 \%$ observed for the whole exome and $88.15 \%$ for chromosome $15\left(\mathrm{P}<2.2 \times 10^{-16}\right.$; Table $\left.\mathrm{I}\right)$. The homozygous variant ratio of chromosome 15 was much higher than the whole exome, which indicate runs of homozygosity for this chromosome.

Homozygosity mapping on chromosome 15 for patient 1. Homozygosity mapping was displayed with HomSI (Fig. 2). Using *.vcf files as an input file, HomSI can identify the majority of homozygous regions. Fig. 2A shows the homozygosity maps for the whole genome, with a continuous blue line observed in patient 1 (first panel), but not in the control individuals (three panels on the right). Fig. 2B-E show the Hom (red) and Het (blue) signal for three control individuals and patient 1 , which was consistent with the homozygosity variant ratio mentioned earlier. In patient 1, the Hom signal was much stronger than the Het signal across the entire chromosome. This indicated the presence of UPD for chromosome 15.

Paternal isodisomy confirmation of patient 1. In order to validate the putative isodisomy, two homozygous variants within chromosome 15 were selected by recommended approaches (11) for Sanger sequencing. The selected variants, namely STARD9 (NM_020759.2:c.4169C>T) and TTBK2 (NM_173500.3:c.2926C>T), were of high quality (read depth, $\geq 10$; genotype quality, $\geq 75$ ) and rarity (1000 Genomes Project; $\mathrm{MAF} \leq 0.001$ and ExAC $\leq 0.001)(11)$. The sequencing results demonstrated that the patient's father was heterozygous for c.4169C $>$ T in gene STARD9 and heterozygous for c. $2926 \mathrm{C}>\mathrm{T}$ in gene $T T B K 2$, whereas both sites on the patient's mother were wild-type with $\mathrm{C}$ genotype (Fig. 3). These results suggested that the patient exhibited paternal UPD.

AS identified by MS-MLPA in patient 2. Patient 2 demonstrated similar karyotype and phenotypes to patient 1 . Suspected AS for patient 2 was validated by another type of efficient genetic test, namely MS-MLPA, which is able to identify CNVs on related regions and the methylation status of imprinted genes. Two ratio charts were created using Coffalyser. Net, based on the MS-MLPA results (Fig. 4). Red dots represent no detected signal in the probes. The MLH-1[HHA1] (Dig) served as a digestion control probe, which exhibited no signal upon digestion. The relative ratio in the $15 q 11-13$ region was $\sim 1$, indicating that no duplication or deletion occurred in this region (Fig. 4A). Furthermore, probes also contained the $U B E 3 A$ gene, which enabled the detection of any deletions on exon 1,2, 3, 4 and 9. Similarly, the ratio was $\sim 1$, suggesting that there were no deletions on exon 1, 2, 3, 4 and 9 of $U B E 3 A$ (Fig. 4A).

Since the imprinted expression gene small nuclear ribonucleoprotein polypeptide $\mathrm{N}(S N R P N)$ is methylated on the maternal, but not the paternal allele (23), the methylation status of MS-MLPA results can be used to discriminate the type of UPD, that is, whether it is paternal or maternal. Five probes were designed for detecting the methylation patterns in the imprinted gene region: NDN-1[HHA1]-419nt, SNRPN-5[HHA1]-250nt, SNRPN-3[HHA1]-178nt, SNRPN-3[HHA1]-190nt and SNRPN-3[HHA1]-142nt. The 
A
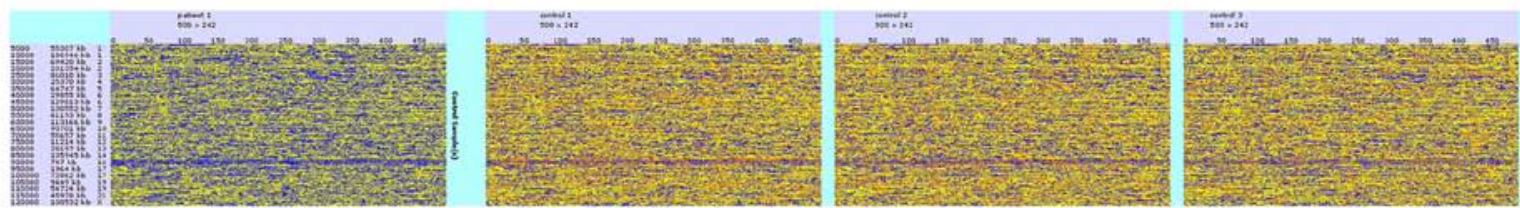

B

Sample: patient 1

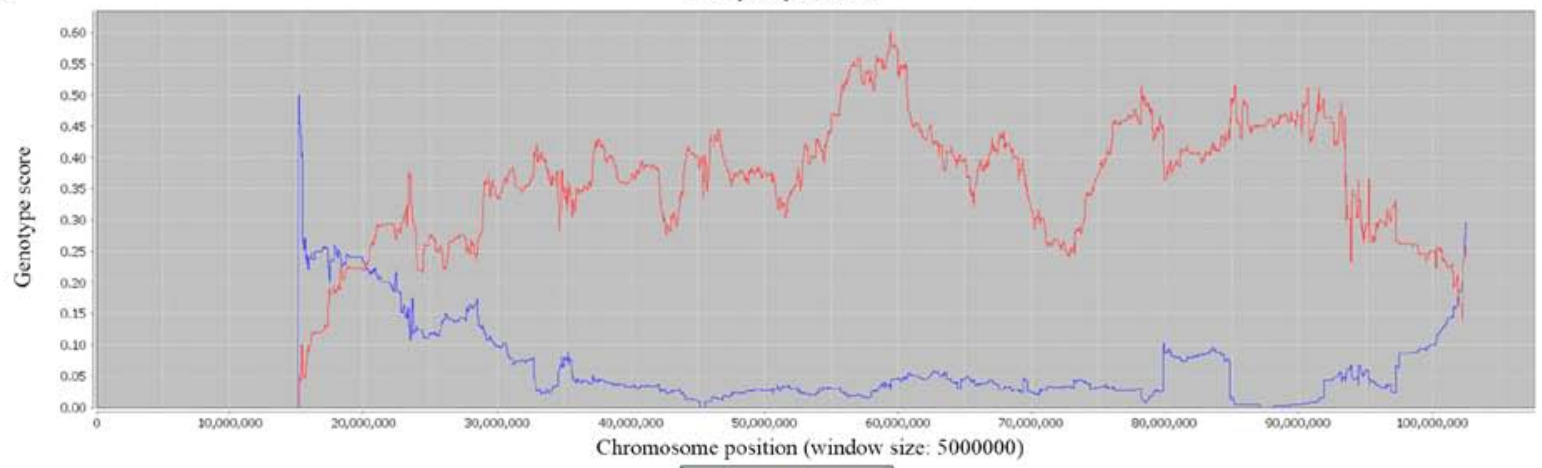

C

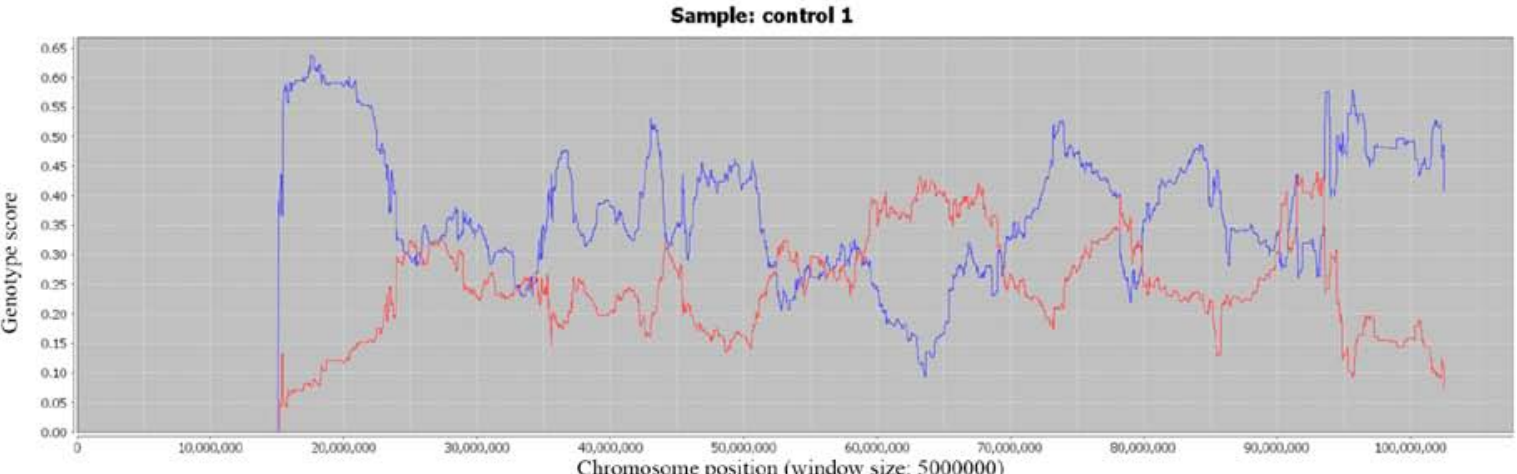

D

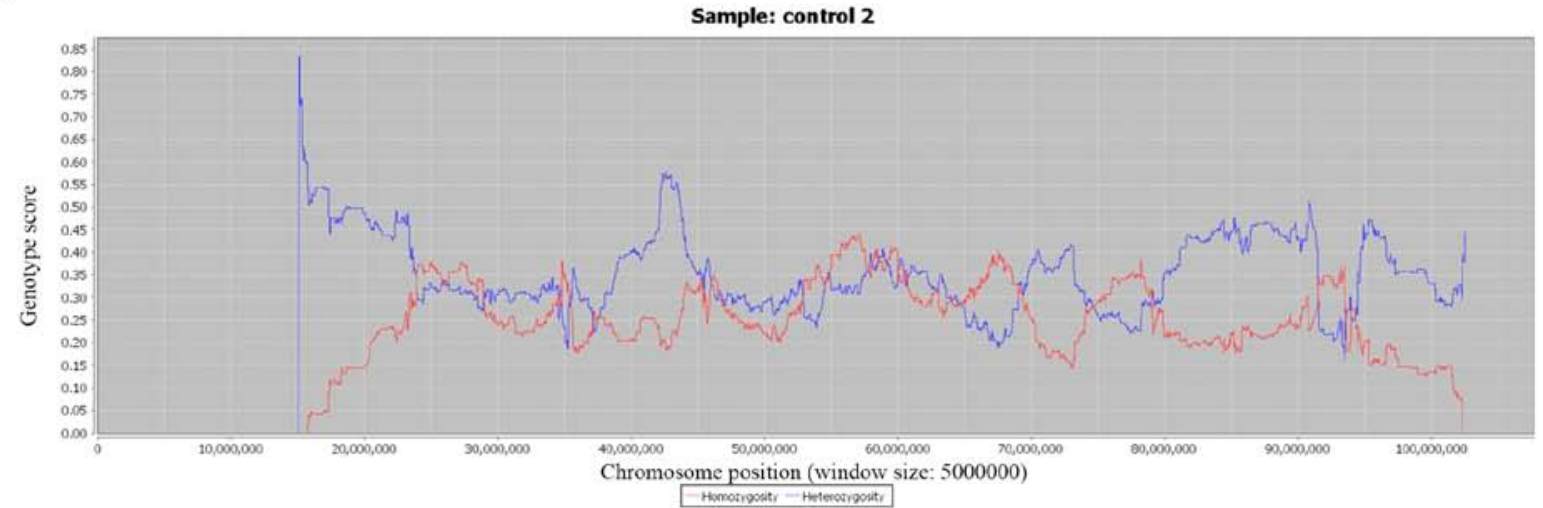

E

Sample: control 3

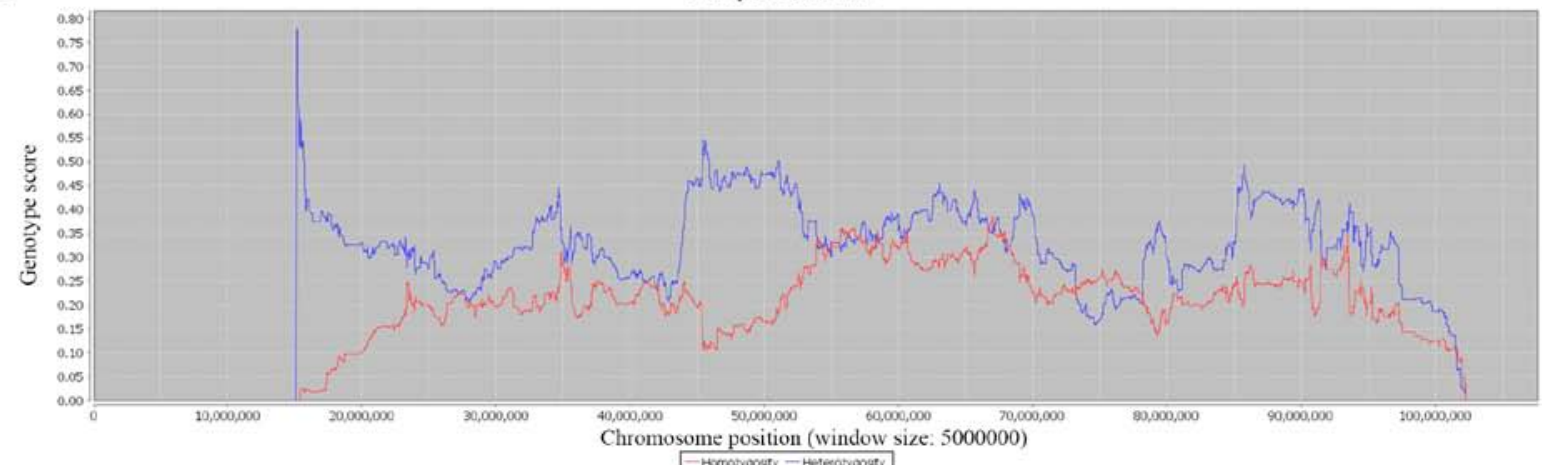

Figure 2. Homozygosity mapping results for patient 1 using the homozygous stretch identifier. (A) Graphical representation of genome-wide homozygosity map for affected (first panel) and unaffected (three panels on the right) individuals. The affected individual was patient 1 , while the unaffected individuals were three healthy controls with normal karyotypes and no inherited disorders. The dashed box indicates runs of shared homozygosity among affected individuals and control individuals. Hom (red) and Het (blue) signals are shown for (B) patient 1, (C) control 1, (D) control 2 and (E) control 3. 
Table I. Results of variants identified by whole exome sequencing.

\begin{tabular}{|c|c|c|c|c|}
\hline \multirow[b]{2}{*}{ Variant type } & \multicolumn{2}{|c|}{ All variants } & \multicolumn{2}{|c|}{$\mathrm{MAF}<0.01$} \\
\hline & Whole exome & Chr15 & Whole exome & Chr 15 \\
\hline Total variants & 63,953 & 1,476 & 18,176 & 470 \\
\hline Homozygous & $42.84 \%$ & $92.69 \%$ & $39.66 \%$ & $88.15 \%$ \\
\hline Synonymous & 11,664 & 254 & 386 & 3 \\
\hline Missense & 11,150 & 236 & 753 & 21 \\
\hline Splice site & 2,845 & 69 & 197 & 3 \\
\hline Frameshift & 384 & 6 & 69 & 2 \\
\hline In-frame coding indel & 390 & 11 & 68 & 1 \\
\hline Stop-gained & 123 & 4 & 31 & 2 \\
\hline Stop-lost & 42 & 0 & 5 & 0 \\
\hline Start-lost & 35 & 1 & 6 & 0 \\
\hline Intron & 32,927 & 725 & 10,011 & 210 \\
\hline Other non-coding & 4,393 & 170 & 6,650 & 228 \\
\hline
\end{tabular}

MAF, minor allele frequency; Chr15, chromosome 15.

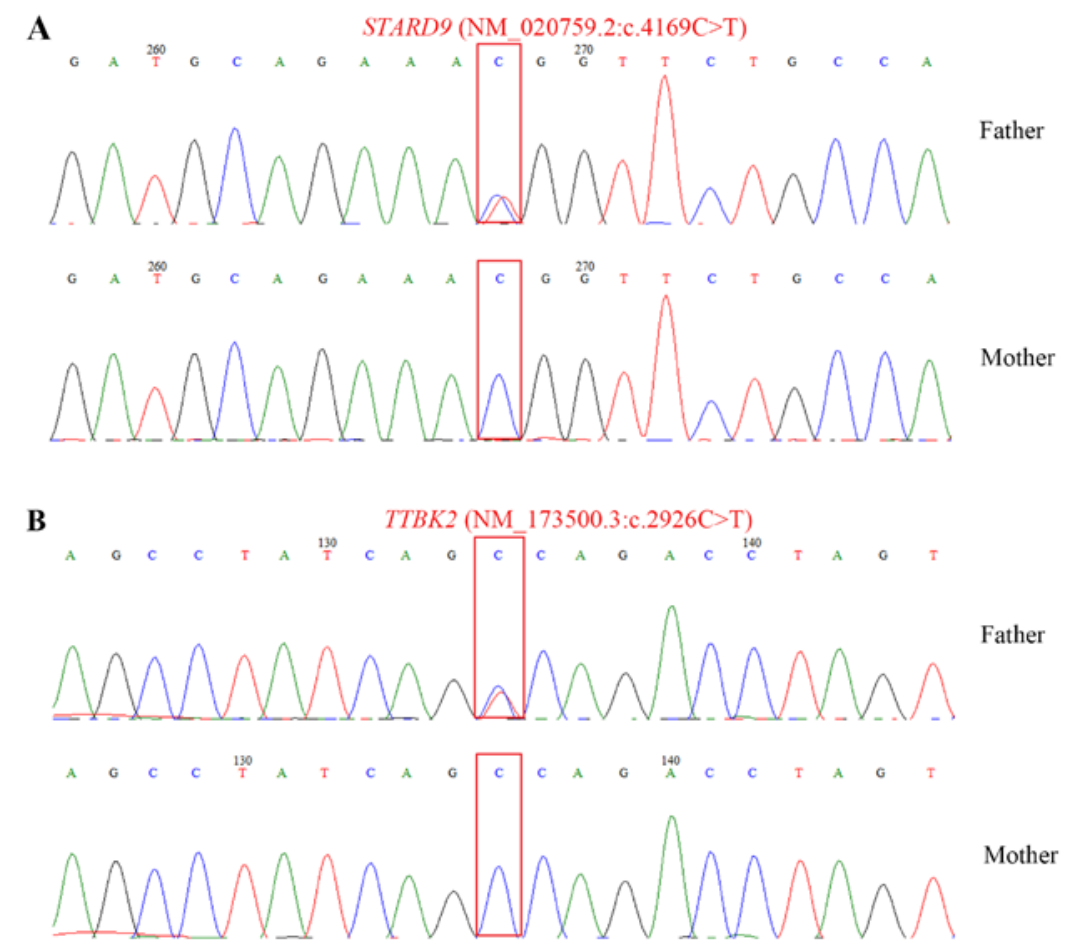

Figure 3. Sanger-based segregation analysis of patient 1. Two homozygous variants (A) STARD9 (NM_020759.2:c.4169C >T) and (B) TTBK2 (NM_173500.3:c.2926C>T), were selected for Sanger-based segregation analysis with available parental DNA. The results indicated that the father is heterozygous for the variants and the mother is homozygous for the wild-type allele, consistent with paternal UPD.

results revealed that no methylation signals were detected on these probes, indicating that patient 2 is highly possible having a paternal UPD (Fig. 4B).

\section{Discussion}

In the present study, two independent cases of suspected AS that was detected by two different testing methods were described. The two patients carried the same karyotype of
Robertsonian-like translocation in chromosome 15. The methods used were both effective in detecting potential UPD, whereas further segregation analysis was required to confirm the parental origin of the disomy. For patient 1, the two selected variants demonstrated that the patient was homozygous at the loci for which the father was heterozygous, suggesting that the structural rearrangement was an isodisomic $15 \mathrm{q}$. A similar approach can be further applied to patient 2 . Since paternal UPD only accounts for $2-3 \%$ of the AS population, 

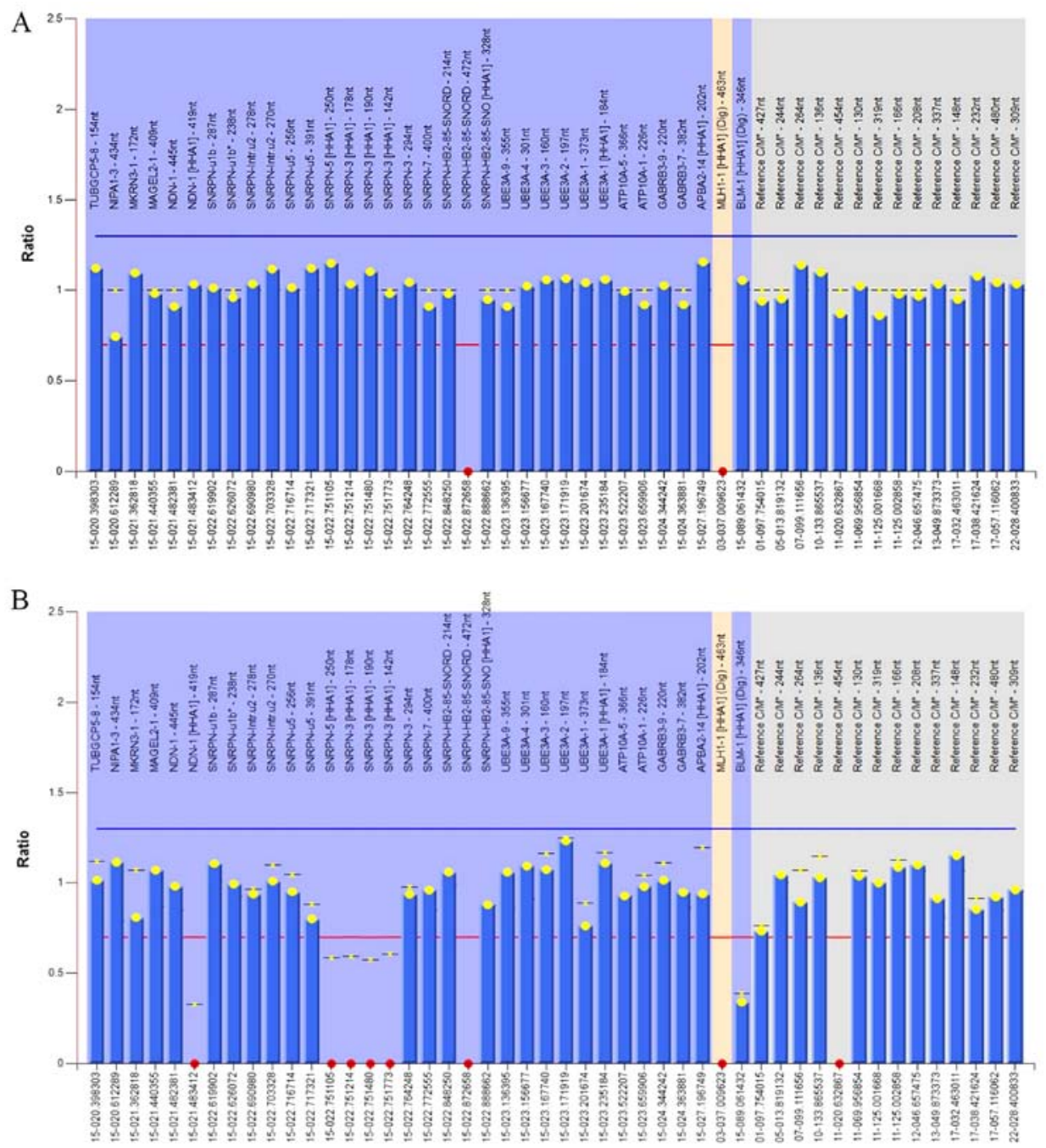

Figure 4. MS-MLPA validation of patient 2. The specifically designed MS-MLPA assay can detect copy number variations and the methylation status of associated genes. A healthy individual served as a reference. Red dots represent no detected signal in the probes. The MLH-1[HHA1] (Dig) served as a digestion control probe, which exhibited no signal upon digestion. (A) The relative ratio in the 15q11-13 region was $\sim 1$, indicating no duplication or deletion (B) No methylation signals were detected on the five probes in the imprinted gene region. The five probes designed for detecting the methylation patterns in the imprinted gene region were as follows: NDN-1[HHA1]-419nt, SNRPN-5[HHA1]-250nt, SNRPN-3[HHA1]-178nt, SNRPN-3[HHA1]-190nt and SNRPN-3[HHA1]-142nt. MS-MLPA, methylation-specific multiplex ligation-dependent probe amplification.

similar cases of AS resulting from isodisomic $15 \mathrm{q}$ associated UPD would be very rare. Currently, the majority of reports on paternal UPD-associated AS cases are heterodisomic (24-27), and cases due to isodisomy are limited $(10,28,29)$.

The severity of AS symptoms varies greatly with mutation types. It has been suggested that AS patients with UPD at chromosome 15 exhibit milder symptoms compared with those with other underlying genetic abnormalities, such as deletions $(28,30)$. As for the two cases reported in the present study, less ataxia and seizures, and better development were observed, which supports previous findings. Varela et al (31) and Williams et al (32) suggested that AS patients with UPD may remain undiagnosed due to their less typical phenotype leading to an under-diagnosis state. WES is a promising approach for increasing the diagnosis rate. Currently, WES is widely used in clinical practice. As compared with whole genome sequencing, exome sequencing displays efficient and effective applications in detecting Mendelian diseases caused by genetic defects, and may further provide therapeutic implications for certain cases $(33,34)$. WES is able to detect SNVs, indels, isodisomic
UPD and even CNVs (35) in a single test, which has been widely accepted as a robust and cost-effective approach. In suspected AS patients, DNA methylation analysis is typically the first test performed, since individuals with AS caused by a 5 -to $7-\mathrm{Mb}$ deletion of 15q11.2-q13, UPD or an imprinting defect only have an unmethylated (i.e. 'paternal') contribution.

It should be noted that the two methods used in the current study have limitations in the diagnosis of AS.MLPA/MS-MLPA can identify maternal deletions and imprinting defects, including paternal UPD (both isodisomy and heterodisomy); however, it is unable to detect point mutations and indels. WES of the proband can detect small nucleotide pathogenic variants in the UBE3A gene, uniparental isodisomy and certain large deletions. However, segregation studies of parental samples are required to determine the parental origin of the affected chromosomes. Furthermore, WES is unable to reliably detect deletions, and does not detect heterodisomy unless parental and grandparental samples are analyzed. Therefore, WES may not be more suitable than MS-MLPA for clinical genetic testing of AS. However, WES can identify most types of 
genetic abnormalities, which is particularly effective for cases with mild or atypical symptoms. Thus, with the advance of sequencing technology, WES would be more suitable and cost-effective in the near future for clinical genetic testing.

In conclusion, the current study suggested the application of WES data in analyzing clinical cases, even if the suspected UPD.

\section{Acknowledgements}

The authors would like to thank Dr. Yongchu Liu and Dr. Yang Liu [Aegicare (Shenzhen) Technology Co., Ltd., Shenzhen, China] for their technical and scientific advice.

\section{Funding}

The present study was supported by The Science and Technology Development Plan of Zhengzhou, 2014 (International Science and Technology Cooperation; grant no. 20140962) and the Science and Technology Development Plan of Zhengzhou, 2014 (grant no. 141PGJHZ530).

\section{Availability of data and materials}

All data generated or analyzed during this study are included in this published article.

\section{Authors' contributions}

HL, HY and QS collected the clinical cases and designed the study. HL and HY performed the analysis and wrote the manuscript. NL performed the MS-MLPA analysis for patient 2. CM and JL prepared the figures and revised the manuscript. QS revised the manuscript and provided instructions for the entire study. All authors read and approved the final manuscript.

\section{Ethics approval and consent to participate}

The present study was approved by the Ethics Committees of the Children's Hospital Affiliated to Zhengzhou University. The two cases involved patients admitted at the Children's Hospital Affiliated to Zhengzhou University.

\section{Patient consent for publication}

Informed consent was obtained from both set of parents for routine and investigative studies.

\section{Competing interests}

The authors declare that they have no competing interests.

\section{References}

1. Kishino T, Lalande M and Wagstaff J: UBE3A/E6-AP mutations cause Angelman syndrome. Nat Genet 15: 70-73, 1997.

2. Rougeulle C, Glatt $\mathrm{H}$ and Lalande M: The Angelman syndrome candidate gene, UBE3A/E6-AP, is imprinted in brain. Nat Genet 17: 14-15, 1997.

3. Sato M: Early origin and evolution of the angelman syndrome ubiquitin ligase gene Ube3a. Front Cell Neurosci 11: 62, 2017.
4. Greer PL,Hanayama R, Bloodgood BL, Mardinly AR,Lipton DM, Flavell SW, Kim TK, Griffith EC, Waldon Z, Maehr R, et al: The angelman syndrome protein Ube3A regulates synapse development by ubiquitinating arc. Cell 140: 704-716, 2010.

5. Clayton-Smith J and Laan L: Angelman syndrome: A review of the clinical and genetic aspects. J Med Genet 40: 87-95, 2003.

6. Fridman C and Koiffmann CP: Origin of uniparental disomy 15 in patients with Prader-Willi or Angelman syndrome. Am J Med Genet 94: 249-253, 2000.

7. Knoll JH, Glatt KA, Nicholls RD, Malcolm S and Lalande M: Chromosome 15 uniparental disomy is not frequent in Angelman syndrome. Am J Hum Genet 48: 16-21, 1991.

8. Siegel DH and Slavotinek A: Uniparental disomy. Pediatr Dermatol 22: 482-487, 2005.

9. Shaffer LG: Risk estimates for uniparental disomy following prenatal detection of a nonhomologous Robertsonian translocation. Prenat Diagn 26: 303-307, 2006.

10. Poyatos D, Guitart M, Gabau E, Brun C, Mila M, Vaquerizo J and Coll MD: Severe phenotype in Angelman syndrome resulting from paternal isochromosome 15. J Med Genet 39: E4, 2002.

11. Bis DM, Schule R, Reichbauer J, Synofzik M, Rattay TW, Soehn A, de Jonghe P, Schöls L and Züchner S: Uniparental disomy determined by whole-exome sequencing in a spectrum of rare motoneuron diseases and ataxias. Mol Genet Genomic Med 5: 280-286, 2017.

12. Carmichael H, Shen Y, Nguyen TT, Hirschhorn JN and Dauber A: Whole exome sequencing in a patient with uniparental disomy of chromosome 2 and a complex phenotype. Clin Genet 84: 213-222, 2013.

13. Kurth I, Baumgartner M, Schabhuttl M, Tomni C, Windhager R, Strom TM, Wieland T, Gremel K and Auer-Grumbach M: Whole exome sequencing in congenital pain insensitivity identifies a novel causative intronic NTRK1-mutation due to uniparental disomy. Am J Med Genet B Neuropsychiatr Genet 171: 875-878, 2016.

14. Gormez Z, Bakir-Gungor B and Sagiroglu MS: HomSI: A homozygous stretch identifier from next-generation sequencing data. Bioinformatics 30: 445-447, 2014.

15. $\mathrm{Li} \mathrm{H}$ and Durbin R: Fast and accurate short read alignment with Burrows-Wheeler transform. Bioinformatics 25: 1754-1760, 2009.

16. Li H, Handsaker B, Wysoker A, Fennell T, Ruan J, Homer N, Marth G, Abecasis G and Durbin R; 1000 Genome Project Data Processing Subgroup: The sequence Alignment/Map format and SAMtools. Bioinformatics 25: 2078-2079, 2009.

17. Talevich E, Shain AH, Botton T and Bastian BC: CNVkit: Genome-Wide copy number detection and visualization from targeted DNA sequencing. PLoS Comput Biol 12: e1004873, 2016.

18. McLaren W, Gil L, Hunt SE, Riat HS, Ritchie GR, Thormann A, Flicek $\mathrm{P}$ and Cunningham F: The ensembl variant effect predictor. Genome Biol 17: 122, 2016.

19. Ng PC and Henikoff S: SIFT: Predicting amino acid changes that affect protein function. Nucleic Acids Res 31: 3812-3814, 2003.

20. Adzhubei IA, Schmidt S, Peshkin L, Ramensky VE, Gerasimova A, Bork P, Kondrashov AS and Sunyaev SR: A method and server for predicting damaging missense mutations. Nat Methods 7: 248-249, 2010

21. Stenson PD, Mort M, Ball EV, Shaw K, Phillips A and Cooper DN: The human gene mutation database: Building a comprehensive mutation repository for clinical and molecular genetics, diagnostic testing and personalized genomic medicine. Hum Genet 133: 1-9, 2014.

22. Danecek P, Auton A, Abecasis G, Albers CA, Banks E, DePristo MA, Handsaker RE, Lunter G, Marth GT, Sherry ST, et al: The variant call format and VCFtools. Bioinformatics 27: 2156-2158, 2011.

23. Glenn CC, Driscoll DJ, Yang TP and Nicholls RD: Genomic imprinting: Potential function and mechanisms revealed by the Prader-Willi and Angelman syndromes. Mol Hum Reprod 3: 321-332, 1997.

24. Tonk V, Schultz RA, Christian SL, Kubota T, Ledbetter DH and Wilson GN: Robertsonian (15q;15q) translocation in a child with Angelman syndrome: Evidence of uniparental disomy. Am J Med Genet 66: 426-428, 1996.

25. Freeman SB, May KM, Pettay D, Fernhoff PM and Hassold TJ: Paternal uniparental disomy in a child with a balanced $15 ; 15$ translocation and Angelman syndrome. Am J Med Genet 45: 625-630, 1993.

26. Fridman C, Varela MC, Nicholls RD and Koiffmann CP: Unusual clinical features in an Angelman syndrome patient with uniparental disomy due to a translocation 15q15q. Clin Genet 54: 303-308, 1998. 
27. Ramsden S, Gaunt L, Seres-Santamaria A and Clayton-Smith J: A case of Angelman syndrome arising as a result of a de novo Robertsonian translocation. Acta Genet Med Gemellol (Roma) 45: 255-261, 1996.

28. Horvath E, Horvath Z, Isaszegi D, Gergev G, Nagy N, Szabó J, Sztriha L, Széll M and Endreffy E: Early detection of Angelman syndrome resulting from de novo paternal isodisomic $15 \mathrm{q}$ UPD and review of comparable cases. Mol Cytogenet 6: 35, 2013.

29. Robinson WP, Christian SL, Kuchinka BD, Peñaherrera MS, Das S, Schuffenhauer S, Malcolm S, Schinzel AA, Hassold TJ and Ledbetter DH: Somatic segregation errors predominantly contribute to the gain or loss of a paternal chromosome leading to uniparental disomy for chromosome 15. Clin Genet 57: 349-358, 2000.

30. Smith A, Robson L and Buchholz B: Normal growth in Angelman syndrome due to paternal UPD. Clin Genet 53: 223-225, 1998.

31. Varela MC, Kok F, Otto PA and Koiffmann CP: Phenotypic variability in Angelman syndrome: Comparison among different deletion classes and between deletion and UPD subjects. Eur J Hum Genet 12: 987-992, 2004.

32. Williams CA, Beaudet AL, Clayton-Smith J, Knoll JH, Kyllerman M, Laan LA, Magenis RE, Moncla A, Schinzel AA, Summers JA and Wagstaff J: Angelman syndrome 2005: Updated consensus for diagnostic criteria. Am J Med Genet A 140: 413-418, 2006.
33. Zhu X, Petrovski S, Xie P, Ruzzo EK, Lu YF, McSweeney KM, Ben-Zeev B, Nissenkorn A, Anikster Y, Oz-Levi D, et al: Whole-exome sequencing in undiagnosed genetic diseases: Interpreting 119 trios. Genet Med 17: 774-781, 2015.

34. Stranneheim $\mathrm{H}$ and Wedell A: Exome and genome sequencing: A revolution for the discovery and diagnosis of monogenic disorders. J Intern Med 279: 3-15, 2016.

35. Pfundt R, Del Rosario M, Vissers LELM, Kwint MP Janssen IM, de Leeuw N, Yntema HG, Nelen MR, Lugtenberg D, Kamsteeg EJ, et al: Detection of clinically relevant copy-number variants by exome sequencing in a large cohort of genetic disorders. Genet Med 19: 667-675, 2017.

This work is licensed under a Creative Commons Attribution-NonCommercial-NoDerivatives 4.0 International (CC BY-NC-ND 4.0) License. 